\title{
Distributed Degenerate Band Edge Oscillator
}

\author{
Ahmed F. Abdelshafy, Dmitry Oshmarin, Mohamed A. K. Othman, Michael M. Green, and Filippo Capolino
}

\begin{abstract}
We propose a new class of oscillators by engineering the dispersion of two-coupled periodic waveguides to exhibit a degenerate band edge (DBE). The DBE is an exceptional point of degeneracy (EPD) of order four, i.e., representing the coalescence of four eigenmodes of a waveguide system without loss and gain. We present a distributed DBE oscillator realized in periodic coupled transmission lines with a unique mode selection scheme that leads to a stable single-frequency oscillation, even in the presence of load variation. The DBE oscillator potentially leads to a boost of the efficiency and performance of RF sources, due to the unique features associated to the EPD concept. This class of oscillators is promising for improving discrete-distributed coherent sources and can be extended to radiating structures to achieve a new class of active integrated antenna arrays.
\end{abstract}

Index Terms-Coupled transmission line, dispersion engineering, degenerate band edge, RF oscillator, ladder oscillator

\section{INTRODUCTION}

O SCILLATORS are one of the fundamental components that exist in any radio frequency (RF) system. Typically, an RF oscillator is an amplifier with positive feedback mechanism utilizing a gain device with a selective resonance circuit that generates a single tone frequency. The negative conductance, i.e., the gain component, required for positive feedback can be obtained using transistors as in cross-coupled transistor pair [1], or by circuit topologies such as Pierce, Colpitts, and Gunn diode waveguide oscillators [2], [3]. In pursuance of improving the performance of RF and microwave sources, many research avenues are currently being investigated [4], [5], [6], [7], [8]. The focus of this paper is on a new class of oscillators whose architecture features i) a cavity made of a periodic coupled-mode waveguide utilizing a special kind of degeneracy in its dispersion diagram, used as the passive circuit responsible for frequency selection, and ii) a set of distributed active devices incorporated in the cavity that provide the sufficient negative conductance to compensate the losses and thus to start the oscillation.

Generally, electromagnetic guiding structures or resonators are characterized by evolution equations that describe the spatial evolution of their eigenstates (eigenvalues and eigenvectors). We are interested in a very special degeneracy condition that occurs when two or more of these eigenstates coalesce into a single degenerate eigenmode at a certain point in the parameter space [9], [10], [11], [12]. Such points are called

This material is based upon work supported by the Air Force Office of Scientific Research award number FA9550-18-1-0355, and by the National Science Foundation under award NSF ECCS-1711975. The authors are thankful to DS SIMULIA for providing CST Studio Suite that was instrumental in this study.

The authors are with the Department of Electrical Engineering and Computer Science, University of California, Irvine, CA 92697 USA. (e-mail: abdelsha@uci.edu,doshmari@uci.edu,mothman@uci.edu, mgreen@uci.edu, f.capolino@uci.edu). exceptional points of degeneracy (EPD), and the order of the EPD is determined by the number of eigenmodes that coalesce at this point. The dispersion relation of eigenmodes in such a guiding structure that exhibits an EPD of order $n$ has the unique behavior in which $\left(\omega-\omega_{e}\right) \propto\left(\beta-\beta_{e}\right)^{n}$ in the vicinity of EPD, where $\omega$ and $\beta$ are the angular frequency and the propagation constant, respectively, at the EPD they are denoted by the subscript $e$. In lossless waveguides, as in this paper, this unique degenerate dispersion behavior is accompanied by supreme characteristics including the vanishing of the group velocity [13], [14] as well as the dramatic improvement in the local density of states [15] resulting in a robust increase in the loaded quality factor of the structure.

In this paper we focus on the degenerate band edge (DBE), which is a fourth-order EPD manifested at the band edge of a lossless structure. At the DBE four eigenstates coalesce into a degenerate one and the dispersion relation near the DBE is characterized by $\left(1-\omega / \omega_{e}\right) \approx \eta_{e}\left(1-k / k_{e}\right)^{4}$ where $\eta_{e}$ is a dimensionless flatness parameter. A finite-length waveguide made of a finite number $N$ of unit cells forms a Fabry-Perot cavity (FPC) with a resonance very near the DBE frequency, see details in [9], [15], [16], and the field creates a standing wave. The resonance closest to the DBE frequency occurs at $\omega_{r, e}$ and for large $N$ it is approximated by $\omega_{r, e} / \omega_{e} \approx 1-\eta_{e} / N^{4}$. Associated to such DBE resonance, the cavity experiences a field enhancement at its geometrical center which leads to an enhancement in the $Q$-factor and less sensitivity to loads [10]. The motivation behind introducing a DBE-based oscillator is based on previous work related to high power microwave devices; it has shown an enhancement of gain in electron beam devices based on waveguide with a DBE [15], [17] and demonstrated a low starting (threshold) current and a unique threshold scaling with length compared to conventional backward wave oscillators [18], [17].

In this paper, we present an example of a DBE oscillator based on two periodic coupled transmission lines (CTLs) as in one of the configurations proposed in [16] and shown in Fig.1(a). The DBE concept in CTLs was introduced in [19] for antenna minimization application. We first show the dispersion of the coupled waveguide where the DBE occurs at several points in the shown frequency range and beyond. Then, we consider a cavity made of a finite-length CTL where discrete distributed gain is introduced leading to a single-frequency of oscillation. We show the robustness of this new class of oscillators against load variation.

The passive waveguide (before the introduction of distributed gain) consists of two coupled microstrips over a grounded dielectric substrate engineered to exhibit a DBE, as shown in Fig. 1(a). In such a waveguide there are two modal fields that can propagate along the $+z$-direction, and two in the opposite one. Proper coupling among the four modal fields is 
required to exhibit a DBE. The realization of proper coupling is achieved either with proximity fields (inductive/capacitive coupling) or with a physical electric connection as in this paper. For more detailed on the engineering of the proper coupling with an approximate LC model see [16] and references therein. The "corrugation" in TL1 (shown in Fig. 1(a)) is introduced to add another degree of freedom to our design space parameters.

The formulation that describes the field evolution using a coupled transmission line approach is found in [10], [16], assuming a time harmonic evolution $e^{j \omega t}$. It is convenient to define a four-dimensional state vector $\boldsymbol{\Psi}(z)=$ $\left[\begin{array}{ll}\mathbf{V}(z), & \mathbf{I}(z)\end{array}\right]^{T}$, which comprises the voltages $\mathbf{V}(z)=$ $\left[\begin{array}{ll}V_{1}(z), & V_{2}(z)\end{array}\right]^{T}$ and currents $\mathbf{I}(z)=\left[\begin{array}{ll}I_{1}(z), & I_{2}(z)\end{array}\right]^{T}$ in the two lines. The first order differential equations that describe the spatial evolution of the state vector in a uniform segment of the CTL are written as [10]

$$
\partial_{z} \boldsymbol{\Psi}(z)=-j \underline{\mathbf{M}} \boldsymbol{\Psi}(z)
$$

where

$$
\underline{\mathbf{M}}=\left[\begin{array}{cc}
\mathbf{0} & -j \mathbf{Z} \\
-j \mathbf{Y} & \mathbf{0}
\end{array}\right]
$$

is the $4 \times 4$ CTL system matrix, and $\mathbf{Z}$ and $\mathbf{Y}$ are the distributed series impedance and shunt admittance $2 \times 2$ matrices describing the per unit length parameters of the two CTLs [20], [10]. The solution of 1 , assuming a certain boundary condition at $z=z_{0}$, is found as $\boldsymbol{\Psi}\left(z_{1}\right)=\underline{\mathbf{T}}\left(z_{1}, z_{0}\right) \mathbf{\Psi}\left(z_{0}\right)$ where $\underline{\mathbf{T}}\left(z_{1}, z_{0}\right)$ is the transfer matrix given by $\underline{\mathbf{T}}\left(z_{1}, z_{0}\right)=$ $\exp \left(-j\left(z_{1}-z_{0}\right) \underline{\mathbf{M}}\right)$.

The periodic structure depicted in Fig. 1(a) has a unit cell composed of two uniform segments A, B and incorporates an additional coupling matrix due to the coupling microstrip. The transfer matrix of a unit cell is expressed as the product of the individual segments' transfer matrices as $\underline{\mathbf{T}}_{\mathrm{U}}=\underline{\mathbf{T}}_{\mathrm{A}} \underline{\mathbf{T}}_{\mathrm{C}} \underline{\mathbf{T}}_{\mathrm{B}}$, where $\underline{\mathbf{T}}_{\mathrm{A}}$ and $\underline{\mathbf{T}}_{\mathrm{B}}$ are the transfer matrices of segments A and $\mathrm{B}$, and $\underline{\mathbf{T}}_{\mathrm{C}}$ is the coupling matrix representing the physical connection via a microstrip between the two lines.

\section{Distributed Degenerate Band Edge Oscillator}

The CTL shown in Fig. 1 is designed on a grounded substrate with relative dielectric constant $\epsilon_{r}=2.2$, height of $0.508 \mathrm{~mm}$, and with a loss tangent of 0.002 . The complex wavenumber-frequency dispersion diagram shown in Fig. 1(c) is obtained using the finite element method, implemented in CST Studio Suite by DS SIMULIA. The dispersion diagram is constructed by extracting the S-parameters of a four-port unit cell and calculating the eigenmode using the associated transfer matrix based on the method discussed in [16]. The results show that various DBEs occur, at frequencies of 2.75 $\mathrm{GHz}$ and $3.02 \mathrm{GHz}$ at $k d=\pi$, and $5.33 \mathrm{GHz}$ and $5.59 \mathrm{GHz}$ at $k d=0$, where all four coalescing wavenumbers are almost real and equal to each other. Losses prevent the realization of a mathematically perfect DBE [16], which is evident from the non-vanishing imaginary part of the wavenumbers at the DBE frequencies in Fig. 1(c). However the main feature of the four coalescing eigenvectors is still retained as discussed in
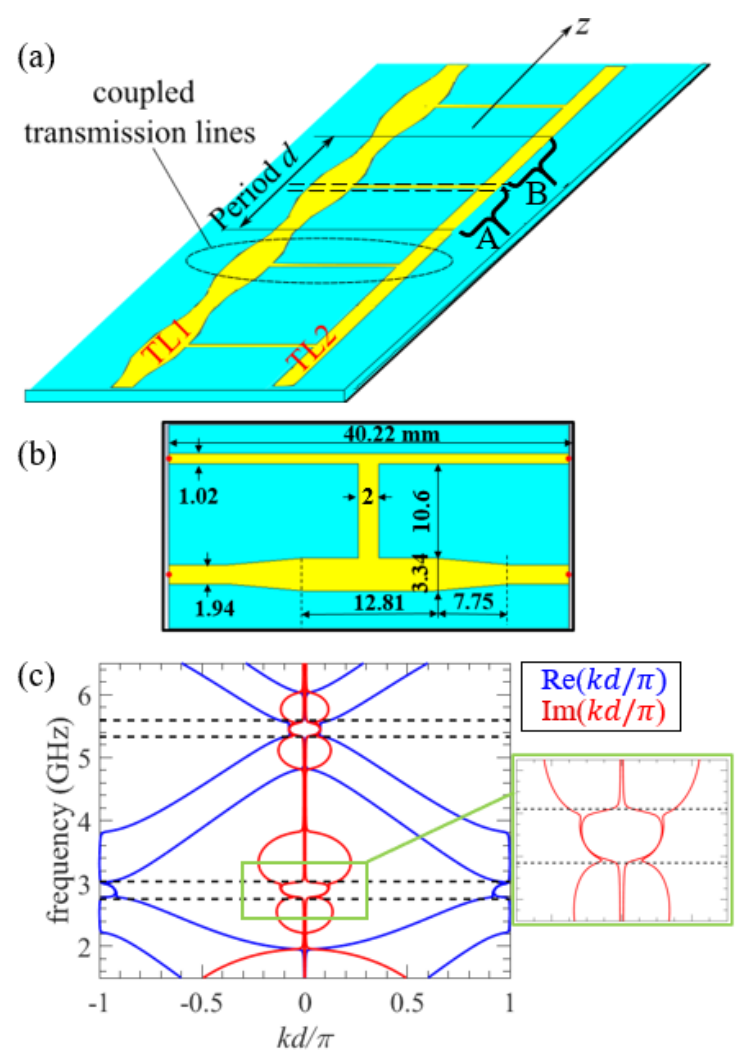

Fig. 1. (a) Schematic of two coupled microstrip lines on a grounded dielectric substrate that support a fourth order EPD (the DBE) visible in the $(k-\omega)$ dispersion diagram. (b) Microstrip unit cell of the periodic waveguide that exhibits the DBE. (c) Real and imaginary parts of the wavenumbers of the four guided Floquet-Bloch modes obtained using the full-wave finite element method accounting for radiation, ohmic and dielectric losses.

[16] using the concept of the eigenvector coalescing parameter (also called hyperdistance).

The distributed DBE oscillator is realized by incorporating discrete active components in a cavity made of a finite-length CTL exhibiting a DBE. Each active component is arranged between two adjacent unit cells to balance small distributed losses and compensate for the two load terminations, and hence start the oscillation as shown in Fig. 2(a). The high quality factor of such DBE cavities and the concept of DBE resonance has been already explained in [18], [21]. Gain is modeled using the non-linear cubic I-V characteristic $i(t)=-g_{m} v(t)+\zeta v^{3}(t)$ of the active device [22] which can be practically implemented with circuits with amplifying devices, such as CMOS transistors or op-amps, with positive feedback. Here $-g_{m}$ is the small-signal slope of the I-V curve in the negative resistance region, and $\zeta$ is the third-order non-linearity constant that models the saturation characteristic of the device. To mimic the clipping mechanism in realistic active devices we choose the turning point (i.e., which is a point in the I-V curve of the active device above which it starts to behave as a passive one; more details are in [22]) $v_{b}=\sqrt{g_{m} / 3 \zeta}$ of the $\mathrm{I}-\mathrm{V}$ characteristics to be 1 volt, and accordingly, we set $\zeta=g_{m} / 3$. The smaller the $\zeta$ value the higher the output swing and the time required to reach steady state. 
In general, active components are realized either as a singleended or differential topology. The most common single-ended realizations are based on two-terminal diodes such as the Gunn diode [23], [24], IMPATT diode [25], and tunnel diode [26]. Such elements are mainly implemented in a compound semiconductor (III-V semiconductor) and used in high power oscillators [23]. On the other hand, a negative differential resistance is most commonly realized in CMOS technology as a cross-coupled transistor pair [1], [7]. As long as the crosscoupled pair is biased by a tail current source, a single-ended version can also be realized, with the other terminal connected to an appropriate dc voltage.

The following calculations and simulations are carried out using the time-domain transient solver implemented in Keysight Advanced Design System (ADS) software by means of scattering parameters obtained through full-wave simulation, where the excitation is modeled by thermal noise generation in the load resistors. The schematic of the proposed distributed oscillator is shown in Fig. 2(a) where the active devices are attached to the lower transmission line in between adjacent unit cells toward the ground (the bias line). For simplicity, we assumed that the gain $-g_{m}$ is equal in all the active devices. In fact, the overall performance of this kind of distributed oscillators can be improved by optimizing the distribution of the gain values of active devices along the finite structure.

To determine the oscillation threshold, which is the minimum value of the gain conductance $g_{m}$ to start oscillations, we tested the finite-length loaded cavity shown in Fig. 2(a) with both ends of the lower TL terminated with $50 \Omega$ and both ends of the upper TL terminated by short circuits to ground. The oscillation threshold is obtained by sweeping the gain $-g_{m}$ value until the oscillation starts. Accordingly, we report that the oscillation threshold is $g_{m, t h}=1 \mathrm{mS}$ for the 8 unit cells oscillator in Fig. 2(a). Note that, the oscillation threshold value depends on the length of the finite structure (i.e. number of unit cells) and the load values $R_{L}$. Therefore, since we analyze the effect of load variation, for the rest of the paper we choose $g_{m}=3 \mathrm{mS}$ to be sufficiently larger than the oscillation threshold $g_{m, t h}$ for a large value of $R_{L}=1 \mathrm{M} \Omega$. The waveform $v_{L}(t)$ at either load reaches a steady state in less than $30 \mathrm{~ns}$ as shown in Fig. 2(b). The oscillation frequency is determined by Fourier transforming $v_{L}(t)$ in the time window from 35 to $100 \mathrm{~ns}$, shown in Fig. 2(c), and it clearly confirms the single-frequency oscillatory behavior despite the length of the cavity and the presence of seven active devices.

Fig. 3 shows the magnitude of voltage and current distributions in the loaded CTL cavity of Fig. 2(a). These voltages and currents are evaluated at nodes $n=1,2, \ldots, 8$, in the presence of the active devices with $g_{m}=3 \mathrm{mS}$. It can be observed that the voltage reaches its peak magnitude in the middle of the cavity; the voltage magnitudes in the lower TL are approximately four times larger than those in the upper TL.

An important advantage of the proposed DBE oscillator is the robustness of the oscillation frequency against a large variation in the load. This robustness is directly related to the strong DBE resonance associated to the cavity made of

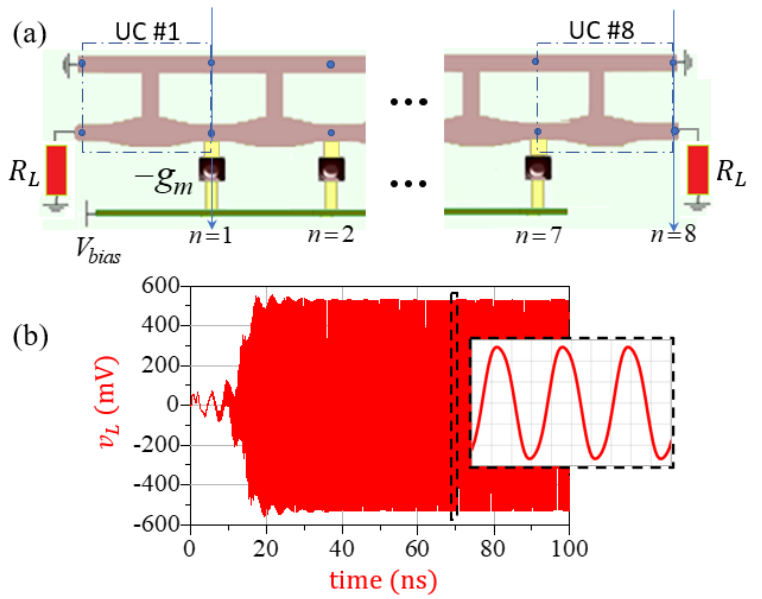

(c)

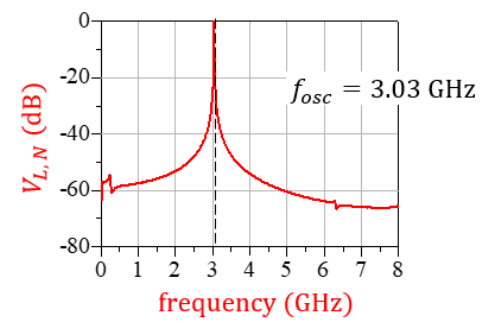

Fig. 2. (a) Loaded DBE oscillator consisting of 8 cascading unit cells (UCs) of microstrip-based CTLs shown in Fig.1(b). Active devices are placed between each two adjacent unit cells to the bias line. Two loads of $50 \Omega$ are attached at the two ends of the lower TL while the upper TL is terminated in short circuits. The oscillation starts for sufficiently large $g_{m}=3 \mathrm{mS}$. (b) Voltage waveform $v_{L}(t)$ monitored at a $50 \Omega$ load where steady state oscillation is observed in less than $30 \mathrm{~ns}$. (c) The normalized spectrum $V_{L, N}(f)$ shows that oscillations occur at $3.03 \mathrm{GHz}$, that is very close to the DBE frequency of $3.02 \mathrm{GHz}$ in Fig. 1(c). The spectrum is calculated by applying the Fourier transform in a time window from 35 to $100 \mathrm{nsec}$.

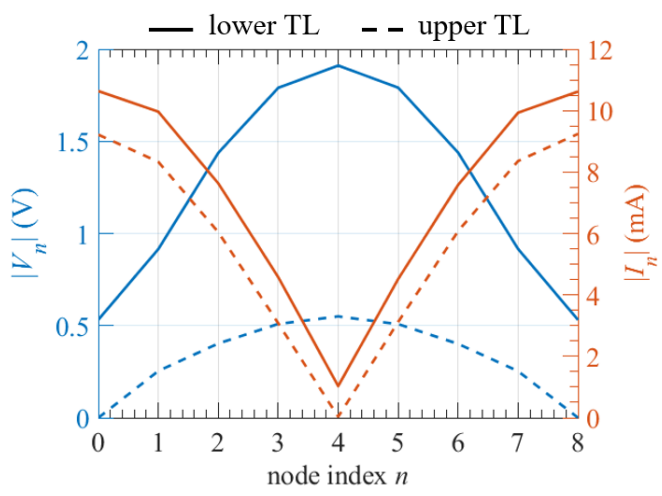

Fig. 3. (a) Voltage and current distributions on top and bottom TLs of the distributed DBE oscillator shown in Fig. 2(a), when the bottom TL is terminated in two $50 \Omega$ loads, while the top TL is short circuited at its two ends. ( $\left|V_{n}\right|$ and $\left|I_{n}\right|$ represent voltage and current time-harmonic waveform peak values, which are phasors' amplitudes, at steady state regime).

a finite-length CTL exhibiting a DBE [9], [15], [22]. This advantage has been shown only for a DBE-based double ladder lumped-element circuit oscillator with only one active device [22]. Typically, the oscillation behavior is very sensitive to the output termination resistance variation resulting in a significant shift in oscillation frequency (e.g. mode jumping in ladder oscillators [4], [27]); in some cases the oscillation stops. Fig. 4 shows the effect of varying the load resistance on 


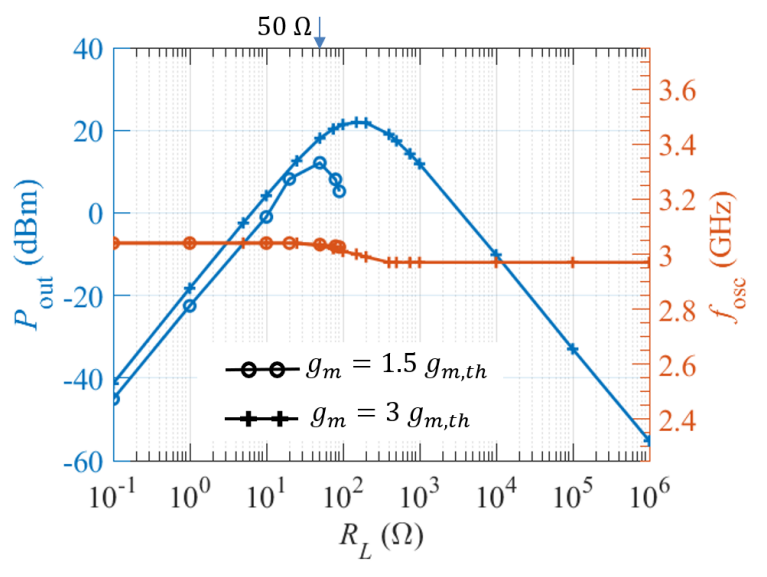

Fig. 4. Average output power and oscillation frequency versus load resistance for the distributed DBE oscillator shown in Fig. 2(a), for $g_{m}=3 \mathrm{mS}$ and $g_{m}=1.5 \mathrm{mS}$. The stability of the oscillation frequency over a huge variation of the load resistance shows an important advantage of the proposed oscillator: the frequency of oscillation is almost the same, i.e. $\sim 3 \mathrm{GHz}$, with a very slight shift that does not exceed $\pm 1 \%(\sim 30 \mathrm{MHz})$.

the oscillation frequency and on the average output power in the proposed distributed DBE oscillator, for $g_{m}=3 \mathrm{mS}$. The result shows a stable frequency of oscillation with a change of only $1 \%$ over a change of load resistance over 7 orders of magnitude. The same plot also shows the total output power on both loads as a function of the load resistance, where the maximum output power corresponds to $R_{L}=150 \Omega$. Note that, the distributed DBE oscillator also shows a stable frequency of oscillation when changing the gain as long as it exceeds the threshold to start the oscillation. This is shown by plotting again the output power and frequency of oscillation for the smaller conductance of $g_{m}=1.5 \mathrm{mS}$. For $g_{m}=1.5 \mathrm{mS}$, the maximum output power occurs when $R_{L}=50 \Omega$ and the oscillation stops when the loads $R_{L} \geq 100 \Omega$ (as $1.5 \mathrm{mS}<$ $\left.g_{m, t h}\right|_{R_{L}=100 \Omega}$ ). Moreover, the robustness of the oscillation frequency against a wide variation in the gain is shown in Fig. 5(a), by fixing the two load resistances to $R_{L}=50 \Omega$ and varying the gain conductance from $g_{m, t h}<g_{m}<9 g_{m, t h}$. It is clear from the red curve in Fig. 5(a) that increasing the gain in the proposed oscillator has a negligible effect on the oscillation frequency (i.e. less than $1 \%$ over the studied range while for $g_{m}>9 g_{m, t h}$ the oscillation frequency jumps to a higher frequency at $7.6 \mathrm{GHz}$ ), whereas the output power (blue curve) increases by increasing the gain conductance. The stability analysis was repeated using a single load resistance, with the other end short circuited, which leads to very similar results and an even high stability of the oscillation frequency over load variation (not reported here for the sake of brevity).

To better understand the robustness of the oscillator to load variations, we add a capacitance $C$ in parallel to each of the two load resistors $R_{L}=50 \Omega$ shown in Fig. 2(a) to imitate the effect of a parasitic capacitance. Fig. 5(b) shows the effect of varying such load capacitance on the oscillation frequency and on the average output power, for $g_{m}=1.5 g_{m, t h}=1.5 \mathrm{mS}$. The variation of the oscillations frequency is negligible for all shown $C$ values.
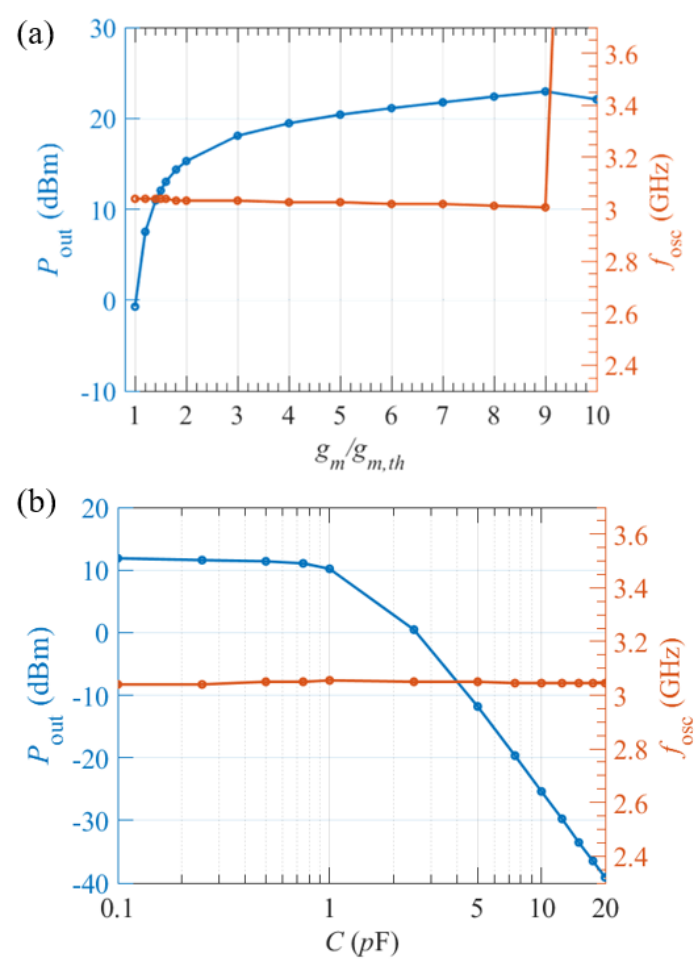

Fig. 5. Average output power and oscillation frequency versus (a) gain variation, and (b) capacitive loads variation. In (b) the capacitor $C$ is parallel to each of the two load resistors $R_{L}=50 \Omega$, assuming $g_{m}=1.5 \mathrm{mS}$.

\section{CONCLUSION}

It has been shown that the DBE in coupled periodic waveguides is useful to conceive new schemes for arrays of coupled oscillators. The DBE structure in the cavity made of a periodic waveguide strongly synchronizes a discrete set of oscillators resulting in an overall single mode of oscillation. The single oscillation frequency, in close proximity to the DBE frequency, has been theoretically demonstrated through fullwave transient simulations. Results demonstrate the stability of the oscillation frequency over a very wide range of load or gain variation, to confirm the stable single-frequency oscillation scheme dictated by the modal degeneracy.

This new scheme of operation is promising for boosting the overall performance of RF sources, where potential benefits include spectral purity and high power efficiency. This scheme based on the DBE concept would be valuable in devices that require several coherent sources, in power combining, with a possible extension to distributed radiating active antenna arrays at microwaves and millimeter waves.

\section{REFERENCES}

[1] B. Razavi, Design of integrated circuits for optical communications. John Wiley \& Sons, 2012.

[2] G. W. Pierce, "Piezoelectric crystal resonators and crystal oscillators applied to the precision calibration of wavemeters," in Proceedings of the American Academy of Arts and Sciences, vol. 59. JSTOR, 1923, pp. 81-106.

[3] R. E. Collin, Foundations for microwave engineering. John Wiley \& Sons, 2007.

[4] T. Endo and S. Mori, "Mode analysis of a multimode ladder oscillator," Circuits and Systems, IEEE Transactions on, vol. 23, no. 2, pp. 100-113, 1976. 
[5] A. Hajimiri and T. H. Lee, "Design issues in CMOS differential LC oscillators," IEEE Journal of Solid-State Circuits, vol. 34, no. 5, pp. 717-724, 1999.

[6] H. Wu and A. Hajimiri, "Silicon-based distributed voltage-controlled oscillators," Solid-State Circuits, IEEE Journal of, vol. 36, no. 3, pp. 493-502, 2001.

[7] B. Razavi and R. Behzad, RF microelectronics. Prentice Hall New Jersey, 1998, vol. 2.

[8] U. L. Rohde, A. K. Poddar, and G. Böck, The design of modern microwave oscillators for wireless applications: theory and optimization. John Wiley \& Sons, 2005.

[9] A. Figotin and I. Vitebskiy, "Gigantic transmission band-edge resonance in periodic stacks of anisotropic layers," Physical Review E, vol. 72 , no. 3 , p. 036619 , Sep. 2005.

[10] M. A. K. Othman and F. Capolino, "Theory of Exceptional Points of Degeneracy in Uniform Coupled-Waveguides and Balance of Loss and Gain," IEEE Transactions on Antennas and Propagation, vol. 65, no. 10, pp. $1-15,2017$.

[11] C. E. Rüter, K. G. Makris, R. El-Ganainy, D. N. Christodoulides, M. Segev, and D. Kip, "Observation of parity-time symmetry in optics," Nature Physics, vol. 6, no. 3, pp. 192-195, 2010.

[12] A. Guo, G. J. Salamo, D. Duchesne, R. Morandotti, M. Volatier-Ravat, V. Aimez, G. A. Siviloglou, and D. N. Christodoulides, "Observation of P T-symmetry breaking in complex optical potentials," Physical Review Letters, vol. 103, no. 9, p. 093902, 2009.

[13] A. Figotin and I. Vitebskiy, "Frozen light in photonic crystals with degenerate band edge," Physical Review E, vol. 74, no. 6, p. 066613, Dec. 2006.

[14] N. Gutman, C. M. de Sterke, A. A. Sukhorukov, and L. C. Botten, "Slow and frozen light in optical waveguides with multiple gratings: Degenerate band edges and stationary inflection points," Physical Review $A$, vol. 85 , no. 3, p. $033804,2012$.

[15] M. A. K. Othman, F. Yazdi, A. Figotin, and F. Capolino, "Giant gain enhancement in photonic crystals with a degenerate band edge," Physical Review B, vol. 93, no. 2, p. 024301, Jan. 2016.

[16] A. F. Abdelshafy, M. A. K. Othman, D. Oshmarin, A. Al-Mutawa, and F. Capolino, "Exceptional Points of Degeneracy in Periodically-Coupled Waveguides and the Interplay of Gain and Radiation Loss: Theoretical and Experimental Demonstration," IEEE Transactions on Antennas and Propagation, vol. 67, no. 09, 2019.

[17] A. F. Abdelshafy, M. A. Othman, F. Yazdi, M. Veysi, A. Figotin, and F. Capolino, "Electron-Beam-Driven Devices With Synchronous Multiple Degenerate Eigenmodes," IEEE Transactions on Plasma Science, vol. 46, no. 8, pp. 3126-3138, 2018.

[18] M. A. Othman, M. Veysi, A. Figotin, and F. Capolino, "Low Starting Electron Beam Current in Degenerate Band Edge Oscillators," IEEE Transactions on Plasma Science, vol. 44, no. 6, pp. 918-929, 2016.

[19] G. Mumcu, K. Sertel, and J. L. Volakis, "Miniature antenna using printed coupled lines emulating degenerate band edge crystals," IEEE Transactions on Antennas and Propagation, vol. 57, no. 6, pp. 16181624, June 2009.

[20] G. Miano and A. Maffucci, Transmission lines and lumped circuits: fundamentals and applications. Academic Press, 2001.

[21] M. Y. Nada, M. A. K. Othman, O. Boyraz, and F. Capolino, "Giant Resonance and Anomalous Quality Factor Scaling in Degenerate Band Edge Coupled Resonator Optical Waveguides," Journal of Lightwave Technology, vol. 36, no. 14, pp. 3030-3039, Jul. 2018.

[22] D. Oshmarin, F. Yazdi, M. A. Othman, J. Sloan, M. Radfar, M. M. Green, and F. Capolino, "New oscillator concept based on band edge degeneracy in lumped double-ladder circuits," IET Circuits, Devices \& Systems, vol. 13, no. 7, pp. 950-957, 2019.

[23] J. B. Gunn, "Microwave oscillations of current in iii-v semiconductors," Solid State Communications, vol. 1, no. 4, pp. 88-91, 1963.

[24] B. Ridley and T. Watkins, "The possibility of negative resistance effects in semiconductors," Proceedings of the Physical Society, vol. 78, no. 2, p. 293, 1961.

[25] W. Shockley, "Negative resistance arising from transit time in semiconductor diodes," Bell System Technical Journal, vol. 33, no. 4, pp. 799-826, 1954.

[26] J. M. Carroll, Tunnel-diode and semiconductor circuits. McGraw-Hill Book Company, 1963.

[27] J. Sloan, M. Othman, and F. Capolino, "Theory of Double Ladder Lumped Circuits With Degenerate Band Edge," IEEE Transactions on Circuits and Systems I: Regular Papers, vol. 65, no. 12, Apr. 2017. 